\title{
New Results on a Visibility Representation of Graphs in 3D
}

\author{
Sándor P. Fekete ${ }^{1}$, Michael E. Houle ${ }^{2}$, Sue Whitesides ${ }^{3} \star \star$ \\ 1 Center for Parallel Computing, Universität zu Köln \\ D-50923 Köln, GERMANY \\ sandorozpr.uni-koeln.de \\ 2 Department of Computer Science, University of Newcastle \\ Callaghan NSW 2308, AUSTRALIA \\ mike@cs.newcastle. edu. au \\ 3 School of Computer Science, McGill University \\ 3480 University St. \#318, Montréal, Québec, CANADA \\ suelopus .mcgill,ca
}

\begin{abstract}
This paper considers a 3-dimensional visibility representation of cliques $K_{n}$. In this representation, the objects representing the vertices are 2-dimensional and lie parallel to the $x, y$-plane, and two vertices of the graph are adjacent if and only if their corresponding objects see each other by a line of sight parallel to the $z$-axis that intersects the interiors of the objects. In particular, we represent vertices by unit discs and by discs of arbitrary radii (possibly different for different vertices); we also represent vertices by axis-aligned unit squares, by axis-aligned squares of arbitrary size (possibly different for different vertices), and by axis-aligned rectangles.

We present:

- a significant improvement (from 102 to 55) of the best known upper bound for the size of cliques representable by rectangles or squares of arbitrary size;

- a sharp bound for the representation of cliques by unit squares $\left(K_{7}\right.$ can be represented but $K_{n}$ for $n>7$ cannot);

- a representation of $K_{n}$ by unit discs.
\end{abstract}

\section{Introduction}

A visibility representation of a graph $G$ maps vertices of $G$ to sets in Euclidean space. An edge $u, v$ occurs in $G$ if and only if the objects representing $u$ and $v$ see each other according to some visibility rule. (In some investigations, the "if and only if" condition is relaxed to "only if".)

* Parts of this work were done while staying at SUNY Stony Brook, supported by NSF Grants ECSE-8857642 and CCR-9204585.

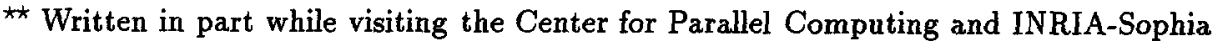
Antipolis. Research supported by NSERC and FCAR. 
Application areas such as VLSI routing and circuit board layout have stimulated considerable research on visibility representations in $R^{2}$. See for example [6], [9], [12], and [13]. Recently, interest has developed in finding good 3dimensional visualizations of graphs. See for example [4], [8], [10]. It is also of interest to develop geometric graph theory in higher dimensions.

This paper continues the study of a particular visibility representation, studied for example in [2], [3], [11] and [1], in which the objects representing vertices are 2-dimensional sets parallel to the $x, y$-plane. An edge $u, v$ occurs in $G$ if and only if the objects representing $u$ and $v$ see each other along a line of sight parallel to the $z$ axis. This line of sight must intersect the interiors of the objects; hence legitimate lines of sight are extensible to tubes of small radius whose ends lie inside the objects. Furthermore, since $G$ has an edge $u, v$ if and only if $u$ and $v$ are mutually visible, the graph $G$ is recoverable from the geometry of the representation. Throughout the paper, we use the term representation to refer to this specific model. Also, when we refer to representation by arbitrary squares or discs, we mean that the size of these objects is allowed to vary with the vertex represented. As in [2] and [3], rectangles are understood to be axis-aligned and disjoint.

In [2] the objects representing vertices are arbitrary rectangles. In this paper, we consider unit squares and discs, and arbitrary squares, rectangles and discs. We focus exclusively on the representation of cliques by these objects.

Constructions for squares can be viewed as constructions for arbitrary rectangles. Any upper bound on the size of the largest clique $K_{n}$ that can be represented by arbitrary rectangles is also an upper bound on the size of the largest $K_{n}$ that can be represented by arbitary squares or by unit squares. The current best bound on the size of the largest $K_{n}$ that can be represented by arbitrary rectangles is 102 (see [2]), which also holds for representation by arbitrary squares and unit squares. Here we lower this bound from 102 to 55 for arbitrary rectangles, and hence for arbitrary squares and unit squares.

The results derived in this paper are as follows:

- $K_{56}$ has no representation by arbitrary rectangles.

- $K_{7}$ (and hence $K_{n}$ for all $n \leq 7$ ) has a representation by unit squares, but no $K_{n}$ for $n \geq 8$ has a representation by unit squares.

- Any $K_{n}$ has a representation by unit discs.

The rest of the paper is organized as follows. Section 2 discusses representations by arbitrary squares and rectangles, Sect. 3 considers representations by unit squares, and Sect. 4 considers representations by arbitrary discs and unit discs.

\section{Squares and Rectangles}

The question of determining the largest complete graph $K_{n}$ that can be represented by arbitrary rectangles is addressed in [2] and [3], which give an upper 
bound of $n \leq 102$ and a lower bound of $n \geq 20$. The construction for $K_{20}$ that appears in [3] uses arbitrary squares. In the following, we describe how to improve the upper bound from $n \leq 102$ to $n \leq 55$.

We consider sequences of $n$ rectangles lying parallel to the $x, y$-plane in $R^{3}$, and ordered by increasing $z$-coordinate. We call a sequence valid if its associated visibility graph is $K_{n}$. Consider the projections of all the rectangles in a valid sequence onto the $x, y$-plane. Because each two must intersect and the objects are axis-aligned rectangles, application of a Helly-type theorem shows that the intersection of all the projections must be non-empty. Thus we can choose a common point $O$ (henceforth regarded as the origin) belonging to the interior of each of the projections. To simplify the notation, we do not distinguish between a rectangle and its projection onto the $x, y$-plane; the meaning will be clear from the context.

Each rectangle $R$ in a valid sequence can be described in terms of the perpendicular distances from $O$ to each of its sides. Instead of giving the $x, y$-coordinates of the vertices of $R$, we describe $R$ as a 4 -tuple $\left(E_{r}, N_{r}, W_{r}, S_{r}\right)$ whose coordinates give, respectively, the distances from $O \in R$ to the east, north, west and south sides of $R$.

We can assume without loss of generality that no two rectangles of a valid sequence share the same value on any of the four coordinates $E, N, W, S$. Hence we can assume that each coordinate value of each of the $n$ rectangles is an integer in the range $[1, n]$ without changing the visibility relationships among the rectangles.

Consider two rectangles $A=\left(E_{a}, N_{a}, W_{a}, S_{a}\right)$ and $B=\left(E_{b}, N_{b}, W_{b}, S_{b}\right)$ in a valid sequence, and denote by $A \cap B$ the intersection of their projections onto the $x, y$-plane. Then $A \cap B$ contains $O$, and the coordinates of $A \cap B$ are $E_{A \cap B}$ $=\min \left\{E_{a}, E_{b}\right\}, N_{A \cap B}=\min \left\{N_{a}, N_{b}\right\}, W_{A \cap B}=\min \left\{W_{a}, W_{b}\right\}$ and $S_{A \cap B}=$ $\min \left\{S_{a}, S_{b}\right\}$. We say that a corner of $A \cap B$ is free if it is not covered by any of the projections of rectangles occuring between $A$ and $B$ in the sequence.

Suppose $A$ and $B$ are rectangles in a valid sequence. Then since $O$ belongs to all the rectangles, at least one of the corners of $A \cap B$ must be free. This is because any rectangle that covers a corner also covers $O$ and hence an entire quadrant of $A \cap B$. Thus if $A \cap B$ had no free corner, it would be covered by the union of the intervening rectangles that cover at least one corner of $A \cap B$.

The northeast corner of $A \cap B$ is not covered by a particular rectangle $R=$ $\left(E_{r}, N_{r}, W_{r}, S_{r}\right)$ between $A$ and $B$ if and only if the Boolean expression $E_{r}<$ $\min \left\{E_{a}, E_{b}\right\}$ OR $N_{r}<\min \left\{N_{a}, N_{b}\right\}$ is true. Similar conditions hold for the other three corners.

We summarize: the rectangles $A$ and $B$. can see each other if and only if one of the following conditions $F$ holds simultaneously for all the rectangles $R$ between $A$ and $B$ :

$\mathbf{F C}_{n e}(A, B)$ : northeast is free, i.e. $\left(E_{r}<\min \left\{E_{a}, E_{b}\right\}\right.$ OR $\left.N_{r}<\min \left\{N_{a}, N_{b}\right\}\right)$;

$\mathbf{F C}_{n w}(A, B):$ northwest is free, i.e. $\left(N_{r}<\min \left\{N_{a}, N_{b}\right\}\right.$ OR $\left.W_{r}<\min \left\{W_{a}, W_{b}\right\}\right)$; 
$\mathbf{F C}_{s w}(A, B):$ southwest is free, i.e. $\left(W_{r}<\min \left\{W_{a}, W_{b}\right\}\right.$ OR $\left.S_{r}<\min \left\{S_{a}, S_{b}\right\}\right)$;

$\mathbf{F C}_{s e}(A, B)$ : southeast is free, i.e. $\left(S_{r}<\min \left\{S_{a}, S_{b}\right\}\right.$ OR $\left.E_{r}<\min \left\{E_{a}, E_{b}\right\}\right)$.

Now we give a definition that is needed in the following discussions. Given a valid sequence of $n$ rectangles $\left(E_{1}, N_{1}, W_{1}, S_{1}\right), \ldots,\left(E_{n}, N_{n}, W_{n}, S_{n}\right)$, we denote by $V_{E}, V_{N}, V_{W}, V_{S}$ the sequences of integers obtained by projecting the rectangles onto their $E, N, W$ and $S$ coordinates, respectively.

The next definition and lemma provide the key tool in our analysis.

Definition 1. A sequence of distinct integers will be called unimaximal if it has exactly one local maximum.

Lemma 2. For all $m>1$, in every sequence of $\left(\begin{array}{c}m \\ 2\end{array}\right)+1$, distinct integers, there exists at least one unimaximal subsequence of length $m$. On the other hand, there exists a sequence of $\left(\begin{array}{c}m \\ 2\end{array}\right)$ distinct integers that has no unimaximal subsequence of length $m$.

This result arises from the Erdös-Szekeres Theorem (1935), whose pigeonhole proof was given by [7]. Lemma 2 is attributed by F. R. K. Chung [5] to V. Chvátal and J. M. Steele, among others.

Lemma 3. In a representation of $K_{5}$ by five rectangles, with no other rectangles present, it is impossible that both sequences $V_{N}$ and $V_{S}$ are unimaximal.

Proof. Suppose both $V_{N}$ and $V_{S}$ were unimaximal. Then $N_{r}>\min \left\{N_{a}, N_{b}\right\}$ and $S_{r}>\min \left\{S_{a}, S_{b}\right\}$ must hold for all rectangles $A, B$, and $R$ between $A$ and $B$. Now consider the conditions $\mathbf{F C}_{n e}, \mathbf{F C}_{n w}, \mathbf{F C}_{s w}, \mathbf{F C}_{s e}$. For $\mathbf{F C}_{n e}(A, B)$ to be true, it must be the case that $E_{R}<\min \left\{E_{a}, E_{b}\right\}$ for all $R$ between $A$ and $B$. The same is true for $\mathbf{F C}_{s e}(A, B)$. Similarly, for $\mathbf{F C}_{n w}(A, B)$ or $\mathbf{F C}_{s w}(A, B)$ to be true, $W_{r}$ must be less than $\min \left\{W_{a}, W_{b}\right\}$. Hence the free corner conditions reduce to the following. One of the two possibilities $\left(W_{r}<\min \left\{W_{a}, W_{b}\right\}\right)$ or $\left(E_{r}<\min \left\{E_{a}, E_{b}\right\}\right)$ holds simultaneously for all rectangles $R$ between $A$ and $B$. This means that all rectangles $A$ and $B$ can see each other along a line of sight with $y$-coordinate 0 . By intersecting the arrangement of five rectangles with the $x, z$-plane, we get an arrangement of 5 line segments in this plane that all see each other. This contradicts the fact that only planar graphs can be represented by vertical visibility of horizontal line segments in a plane. (See [12] and [13] for results on such representations in the plane.)

Theorem 4. No complete graph $K_{n}$ has a representation by arbitrary rectangles for $n \geq 56$.

Proof. Suppose we had a representation of $K_{n}$ with $n \geq 56$. Lemma 2 implies that $V_{N}$ has a unimaximal subsequence $V_{N}^{\prime}$ of length 11 . Consider the associated 

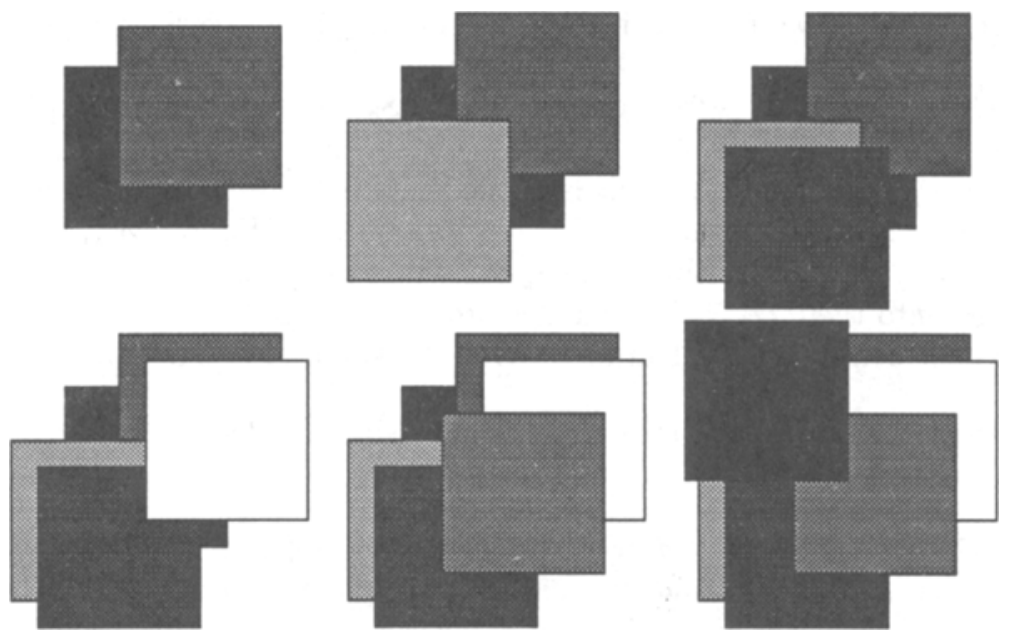

Fig. 1. Representing $K_{7}$ by unit squares.

subsequence $V_{S}^{\prime}$ of length 11 . It follows again from Lemma 2 that there is a subsequence $V_{S}^{\prime \prime}$ of length 5 that is unimaximal. Remove the rectangles not associated with the subsequence. This destroys no visibility lines, so the five remaining rectangles represent $K_{5}$. However, both $V_{S}^{\prime \prime}$ and its corresponding subsequence $V_{N}^{\prime \prime}$ are unimaximal. This contradicts Lemma 3.

\section{Unit Squares}

While it is still a challenge to narrow the gap between the known upper and lower bounds for representation of $K_{n}$ by rectangles or general squares, we will see in the following that the largest possible $n$ for which $K_{n}$ has a representation by squares of equal size is $n=7$.

Theorem 5. $K_{7}$ has a representation by unit squares.

Proof. See Figure 1. The six phases indicate how the seven squares are placed on top of each other.

Theorem $6 . K_{8}$ does not have a representation by unit squares.

Proof. We did the proof by a computer search that enumerates all maximal valid sequences of squares. The search begins with a single square. It proceeds in a depth-first-search manner, examining all the ways to add a new square above the squares in the valid sequence currently considered. It tests validity by checking 
the free corner conditions of the previous section. Whenever a valid extension is discovered, an immediate attempt is made to extend it further.

To be more specific, consider a valid sequence, not necessarily maximal, of $m$ squares of the same "unit" size. As before, without loss of generality, we may describe these squares as 4-tuples $(E, N, W, S)$. The coordinate positions give rise to four sequences $V_{E}, V_{N}, V_{W}, V_{S}$ of distinct integers in the range $[1, m]$. Because all the squares have the same size, the $E$ and $W$ coordinates of any given square must sum to $m+1$, and similarly for the $N$ and $S$ coordinates. Hence $V_{E}$ determines $V_{W}$, and $V_{N}$ determines $V_{S}$. Since the sequence is valid, the free corner conditions given in the previous section must hold for each pair of squares in the sequence.

Now suppose we want to enumerate all the possible ways to position a new unit square above the existing $m$ squares. Each new sequence will be described by 4 -tuples whose coordinates are integers in the range $[1, m+1]$. There are $m+1$ choices for the $E$ coordinate of the new square and $m+1$ choices for its $N$ coordinate. Since these choices determine $W$ and $S$, there are in total $(m+1)^{2}$ possibilities. These may be considered by lexigraphic order of the $(E, N)$ pair of coordinates of the new square, from $(E, N)=(1,1)$ to $(E, N)=(m+1, m+1)$.

Note that although the squares in the old sequence do not change position in any way, their coordinates must change to make room for the coordinates of the new square. For example, if the new square has $E=3$, then all old squares with an $E$ coordinate of 3 or greater must increase the old $E$ coordinate by 1 .

To check whether a new sequence is valid, we need only check the free corner conditions of the previous section for pairs of squares involving the new square.

Our program enumerates all valid maximal sequences of unit squares as follows. It begins with a valid sequence of length $m=1$, described in the $E, N, W, S$ coordinate system by the single 4 -tuple $(1,1,1,1)$. Then it carries out a depthfirst-search as follows. When processing a valid sequence of length $m$, it considers in lexigraphic order the possibilities for adding a new square above the existing ones. If a sequence of length $m+1$ proves to be valid, it is recursively processed immediately.

Since every prefix of a valid sequence is valid, and since the number of valid sequences is finite (recall that $m \leq 55$ ), this search eventually discovers all valid sequences.

Using this depth-first-search strategy, we were able to generate all maximal valid sequences in three minutes running time on a SPARC 1 . There were 2064 of these maximal valid sequences, all of which had length 7 .

\section{Discs}

After the results on rectangles and squares, we examine the situation for discs. We show that any $K_{n}$ can be represented.

Theorem 7. Any $K_{n}$ can be represented by unit discs. 


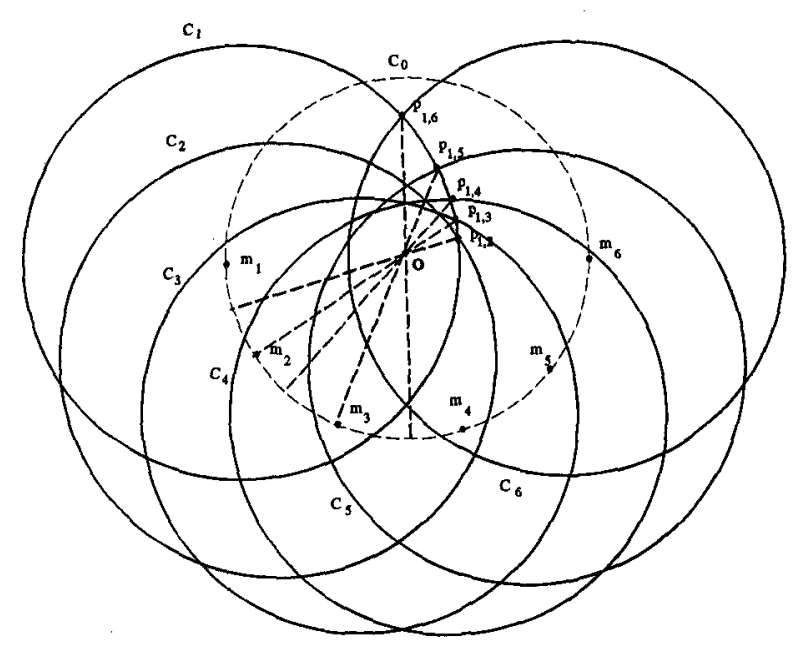

Fig. 2. Representing $K_{n}$ by $n$ unit discs.

Proof. See Figure 2. Let $\frac{1}{2}<r<1$ and consider the circle $c_{0}$ of radius $r$ centered at the origin $O$. Now pick $n$ points $m_{1}=\left(x_{1}, y_{1}, 0\right), \ldots, m_{n}=\left(x_{n}, y_{n}, 0\right)$ equally spaced and in counter-clockwise order on an arc of $c_{0}$ of length less than half the circumference of $c_{0}$. Without loss of generality, assume that all $y_{i}<0$. Let $M_{i}=m_{i}+(0,0, i)$. Let $C_{i}$ be the unit disc parallel to the $x, y$-plane with center $M_{i}$, and let $c_{i}$ be its projection onto the $x, y$-plane. We claim that the $C_{i}$ form a representation for $K_{n}$.

It suffices to show that for each $i, 1 \leq i<n$, all the discs above $C_{i}$ can see $C_{i}$, i.e., $C_{j}$ can see $C_{i}$ whenever $j>i$.

Consider the points $p_{i j}$ obtained by selecting from the two intersection points of the boundaries of $c_{j}$ and $c_{i}$ the one that has positive $y$-coordinate. (Figure 2 illustrates this for $i=1$.) Consider some fixed $i$ and the set of indices $j>i$. Observe that $p_{i j}$ lies on the perpendicular bisector of the line segment $\left[m_{i}, m_{j}\right]$. This bisector runs through 0 . As $j$ increases, so does the angle between the $x$ axis and $\left[0, p_{i j}\right]$. This means that for $j>i$, the $p_{i j}$ appear in counter-clockwise order around the boundary of $c_{i}$. Therefore none of the points $P_{j}=p_{j}+(0,0, j)$ on discs $C_{j}, i<j \leq n$, are blocked from the view of the boundary of disc $C_{i}$. Obviously if $j=i+1$, then $C_{i}$ and $C_{j}$ are mutually visible along lines of sight with $x, y$-projection in $c_{i} \cap c_{i+1}$. Now suppose $j>i+1$. Because $c_{i+1} \cup c_{j-1}$ contains $c_{k}$ for $i+1 \leq k \leq j-1, C_{i}$ and $C_{j}$ are visible along lines of sight with $x, y$-projection in $c_{i} \cap c_{j} \cap c_{i+1}^{\prime} \cap c_{j-1}^{\prime}$, where the prime denotes complement. For $j=i+2$, this figure is bounded by three circular arcs, and for $j>i+2$, by four circular arcs. 
Acknowledgement: We would like to thank Günter Rote for a significant simplification of the proof of Lemma 3 .

\section{References}

1. H. Alt, M. Godau and S. Whitesides. Universal 3-dimensional visibility representations for graphs. See elsewhere in these proceedings.

2. P. Bose, H. Everett, S. Fekete, A.Lubiw, H. Meijer, K. Romanik, T. Shermer and S. Whitesides. On a visibility representation for graphs in three dimensions. Proc. Graph Drawing '93, Paris (Sèvres), 1993, pp. 38-39.

3. P. Bose, H. Everett, S. Fekete, A. Lubiw, H. Meijer, K. Romanik, T. Shermer and S. Whitesides. On a visibility representation for graphs in three dimensions. Snapshots of Computational and Discrete Geometry, v. 3, eds. D. Avis and P. Bose, McGill University School of Computer Science Technical Report SOCS-94.50, July 1994, pp. 2-25.

4. R. Cohen, P. Eades, T. Lin and F. Ruskey. Three-dimensional graph drawing. Proc. Graph Drawing '94, Princeton NJ, 1994, Lecture Notes in Computer Science LNCS \#894, Springer-Verlag, 1995, pp. 1-11.

5. F. R. K. Chung. On unimodal subsequences. J. Combinatorial Theory, Series A, v. 29,1980 , pp. $267-279$.

6. A. Dean and J. Hutchison. Rectangle visibility representations of bipartite graphs. Proc. Graph Drawing '94, Princeton NJ, 1994. Lecture Notes in Computer Science LNCS \#894, Springer-Verlag, 1995, pp. 159-166.

7. J. M. Hammersley. A few seedlings of research. Proc. $6^{\text {th }}$ Berkeley Symp. Math. Stat. Prob., U. of California Press, 1972, pp. 345-394.

8. H. Koike. An application of three-dimensional visualization to object-oriented programming. Proc. of Advanced Visual Interfaces AVI '92, Rome, May 1992, v. 36 of World Scientific Series in Computer Science, 1992, pp. 180-192.

9. E. Kranakis, D. Krizanc and J. Urrutia. On the number of directions in visibility representations of graphs. Proc. Graph Drawing '94, Princeton NJ, 1994, Lecture Notes in Computer Science LNCS \#894, Springer-Verlag, 1995, pp. 167-176.

10. J. Mackinley, G. Robertson and S. Card. Cone trees: animated 3d visualizations of hierarchical information. Proc. of the SIGCHI Conf. on Human Factors in Computing, 1991, pp. 189-194.

11. K. Romanik. Directed VR-representable graphs have unbounded dimension. Proc. Graph Drawing '94, Princeton, NJ, 1994, Lecture Notes in Computer Science LNCS \#894, Springer-Verlag, 1995, pp. 177-181.

12. R. Tamassia and I. Tollis. A unifed approach to visibility representations of planar graphs. Discrete Comput. Geom. v. 1, 1986, pp. 321-341.

13. S. Wismath. Characterizing bar line-of-sight graphs. Proc. ACM Symp. on Computational Geometry, 1985, pp. 147-152. 